\title{
THE FILLING RADIUS OF TWO-POINT HOMOGENEOUS SPACES
}

\author{
MIKHAIL KATZ
}

Let $X$ be a closed connected manifold of dimension $n$, and let dist $=$ $\operatorname{dist}\left(x, x^{\prime}\right)$ be a Riemannian metric on $X$. The function $d_{x}$ on $X$ given by $d_{x}\left(x^{\prime}\right)=\operatorname{dist}\left(x, x^{\prime}\right)$ lies in the space $L^{\infty}(X)$ of all bounded functions on $X$ with the sup-norm \|\| . The canonical inclusion

$$
X \rightarrow L^{\infty}(X), \quad x \mapsto d_{x}
$$

is an isometric imbedding, as $\operatorname{dist}\left(x, x^{\prime}\right)=\left\|d_{x}-d_{x^{\prime}}\right\|$ (the triangle inequality). Consider the inclusion homomorphism $\alpha_{\varepsilon}: H_{n}(X) \rightarrow H_{n}\left(U_{\varepsilon} X\right)$, where $U_{\varepsilon} X \subset$ $L^{\infty}(X)$ is the $\varepsilon$-neighborhood of $X$, and the coefficients are in $\mathbf{Z}_{2}$. Following M. Gromov [3], we introduce a new metric invariant of $X$.

Definition. The filling radius of $X$, denoted Fill $\operatorname{Rad} X$, is the infimum of those $\varepsilon>0$ for which $\alpha_{\varepsilon}([X])=0$, where $[X]$ is the fundamental class of $X$.

We prove the following theorems.

Theorem 1. The filling radius of the real projective space $\mathbf{R} P^{n}$ of constant curvature +1 equals one third of its diameter:

$$
\text { Fill } \operatorname{Rad} \mathbf{R} P^{n}=\frac{1}{3} \operatorname{diam} \mathbf{R} P^{n}=\frac{\pi}{6} .
$$

Theorem 2. The filling radius of the sphere $S^{n}$ of constant curvature +1 equals one half of the spherical distance between two vertices of an inscribed regular $(n+1)$-simplex:

$$
\text { Fill } \operatorname{Rad} S^{n}=\frac{1}{2} \arccos \left(-\frac{1}{n+1}\right)
$$

We also obtain partial results for the projective spaces over the complex numbers, the quaternions, and the Cayley numbers (see Propositions 1-3). Our estimates from below for the filling radius of two-point homogeneous spaces depend on a version of Jung's theorem (see Lemma 2) and the AlexandrovToponogov comparison theorem (see Lemma 3).

The author would like to thank Professor Gromov for posing the problem and for his much-needed guidance throughout the work on this article.

Received July 30, 1982, and, in revised form, April 6, 1983. 


\section{Estimates from above}

Lemma 1. Suppose a manifold $X$ contains a closed subset $Y$ such that, for some $R>0$, the following two conditions are satisfied:

(i) $\operatorname{diam} Y \leqslant 2 R$,

(ii) $\operatorname{dist}(x, Y) \leqslant 2 R$ for every point $x \in X$.

Then Fill $\operatorname{Rad} X \leqslant R$.

Proof. We will extend the canonical imbedding $X \rightarrow L^{\infty}(X), x \mapsto d_{x}$ to a mapping of the cone over $X$. The function $f \in L^{\infty}(X)$ given by $f(x)=$ $\operatorname{dist}(x, Y)+R$ in view of condition (i) satisfies

(iii) $\left\|f-d_{y}\right\|=R$ for all $y \in Y$.

We join $d_{x}$ with $f$ by a path $\left\{d_{x}^{t}\right\} \subset L^{\infty}(X)$ defined for $t \geqslant 0$ as follows. The value of $d_{x}$ at a given point $z \in X$ moves toward $f(z)$ with unit speed, and having reached it, stops and changes no more, or analytically

$$
d_{x}^{t}(x)= \begin{cases}\max \left(f(z), d_{x}(z)-t\right) & \text { if } d_{x}(z) \geqslant f(z) \\ \min \left(f(z), d_{x}(z)+t\right) & \text { if } d_{x}(z)<f(z)\end{cases}
$$

Properties (ii) and (iii) imply $d_{x}^{3 R}=f$ for $t=3 R$. In other words, the functions $d_{x}^{t}$, where $x \in X, t \in[0,3 R]$, form a topological cone (imbedded in $L^{\infty}(X)$ with possible self-intersections) which retracts $X \subset L^{\infty}(X)$ to the point $f \in$ $L^{\infty}(X)$.

To prove the lemma, it suffices to show that for each function $d_{x}^{t}$ there exists a point $x^{\prime} \in X$ such that $\left\|d_{x}^{t}-d_{x^{\prime}}\right\| \leqslant R$. We have $\left\|d_{x}^{t}-d_{x}\right\| \leqslant R$ for $t \leqslant R$, by definition of $d_{x}^{t}$. We will show that $\left\|d_{x}^{t}-d_{y}\right\|=R$ for $R \leqslant t \leqslant 3 R$, if $y \in Y$ and $\operatorname{dist}(x, y) \leqslant 2 R$.

Let $t=R$. Then we show that

(iv) $\left\|d_{x}^{R}-d_{y}\right\|=R$ if $\operatorname{dist}(x, y) \leqslant 2 R$.

Indeed, if $\left|d_{x}(z)-f(z)\right| \leqslant R$ for some point $z \in X$, then $d_{x}^{R}(z)=f(z)$ and hence $\left|d_{x}^{R}(z)-d_{y}(z)\right| \leqslant R$ by (iii). If $\left|d_{x}(z)-f(z)\right|>R$, then in view of (iii) the number $d_{x}(z)$ does not lie between $d_{y}(z)$ and $f(z)$. Since $\left|d_{x}(z)-d_{y}(z)\right| \leqslant$ $2 R$, the definition of $d_{x}^{t}$ implies that $\left|d_{x}^{R}(z)-d_{y}(z)\right| \leqslant R$.

Finally, condition (ii) gives a point $y \in Y$ with $\operatorname{dist}(x, y) \leqslant 2 R$, and properties (iii) and (iv) show that $\left\|d_{x}^{t}-d_{y}\right\| \leqslant R$ for $R \leqslant t \leqslant 3 R$.

Corollary. The filling radius of every Riemannian manifold is less than or equal to one third of its diameter.

Proof. The ball of radius $\frac{1}{3}$ diam $X$ centered at any point of the manifold $X$ satisfies the conditions of Lemma 1 with $R=\frac{1}{3}$ diam $X$.

Proof of Theorem 1. Apply the preceding corollary together with [3, Lemma 1.2.B]. 
Remarks. 1. If an arbitrary metric space $X$ contains a subset $Y$ satisfying both conditions of Lemma 1 , then the cone defined by $(+)$ retracts $X$ to a point in the closed $R$-neighborhood of $X \subset L^{\infty}(X)$.

2. One can define Riemannian metrics $g$ on $\mathbf{R} P^{2}$ close to the constant curvature metric, for which $\operatorname{Fill} \operatorname{Rad}\left(\mathbf{R} P^{2}, g\right)=\frac{1}{3}$ diam $g$. In such case, every point of $\left(\mathbf{R} P^{2}, g\right)$ lies on some closed geodesic of length 2 diam $g$. In general, as it will be shown elsewhere, every Riemannian manifold $X$ satisfying the extremal equality Fill $\operatorname{Rad} X=\frac{1}{3} \operatorname{diam} X$ has a geodesic loop of length 2 diam $X$ at every point.

\section{Jung's theorem and the filling radius of spheres}

Let $S^{n}$ be the unit sphere in $\mathbf{R}^{n+1}$ with distance between two points measured along a shortest spherical arc joining them, and $S^{n} E$ the unit sphere with the Euclidean metric defined by the length of the line interval in $\mathbf{R}^{n+1}$ joining two points of the sphere. Let $L\left(S^{n}\right)$ (respectively, $L\left(S^{n} E\right)$ ) be the spherical (respectively, Euclidean) distance between two vertices of a regular $(n+1)$-simplex inscribed in the unit sphere. We also denote by $l\left(S^{n}\right)$ and $l\left(S^{n} E\right)$ the respective distances between the point of the sphere representing the center of some $n$-face of the simplex, and any vertex of this $n$-face. We have

$$
\begin{aligned}
L\left(S^{n}\right) & =\arccos (-1(n+1)), \quad L\left(S^{n} E\right)=\sqrt{2+2 /(n+1)}, \\
l\left(S^{n}\right) & =\arccos (1 /(n+1))=\pi-L\left(S^{n}\right), \\
l\left(S^{n} E\right) & =\sqrt{2-2 /(n+1)}=\sqrt{4-L\left(S^{n} E\right)^{2}} .
\end{aligned}
$$

Lemma 2 (Jung's theorem). Every subset of $S^{n}$ of diameter $\leqslant L\left(S^{n}\right)$ either coincides with the set of vertices of some inscribed regular $(n+1)$-simplex, or is contained in some ball of radius $l\left(S^{n}\right)$. The same assertion is true with $S^{n}, L\left(S^{n}\right)$, $l\left(S^{n}\right)$ replaced by $S^{n} E, L\left(S^{n} E\right), l\left(S^{n} E\right)$, respectively.

A subset of the unit sphere has spherical diameter $\leqslant L\left(S^{n}\right)$ if and only if it has Euclidean diameter $\leqslant L\left(S^{n} E\right)$, while a ball in $S^{n}$ of radius $l\left(S^{n}\right)$ corresponds to a ball in $S^{n} E$ of radius $l\left(S^{n} E\right)$. Therefore it suffices to prove Jung's theorem in the Euclidean case. For a proof, see [2, p. 200].

Proof of Theorem 2. The set of vertices of a regular $(n+1)$-simplex inscribed in $S^{n}$ satisfies the conditions of Lemma 1 with $2 R=L\left(S^{n}\right)$, hence Fill $\operatorname{Rad} S^{n} \leqslant \frac{1}{2} L\left(S^{n}\right)$. To prove the opposite inequality, suppose that the fundamental class of $S^{n}$ vanishes in the open $\frac{1}{2} L\left(S^{n}\right)$-neighborhood of $S^{n} \subset$ $L^{\infty}\left(S^{n}\right)$. This means (cf. $\left.[3, \S 1.2 . C]\right)$ that $\alpha_{*}\left(\left[S^{n}\right]\right)=0$, where $\alpha: S^{n} \rightarrow T$ is the 
inclusion of $S^{n}$ in some polyhedron $T$ contained in this neighborhood. We will retract $T$ to $S^{n}$ and thus prove the desired inequality.

We map the 0 -skeleton of $T$ to $S^{n}$ by sending each vertex to a nearest point of $S^{n}$. We may assume that $T$ is triangulated into sufficiently small simplices so that the endpoints of each edge of $T$ are sent to points of $S^{n}$ with distance < $L\left(S^{n}\right)$. By Jung's theorem, the set of vertices of every given simplex is sent to some open hemisphere of $S^{n}$ and hence spans a canonical geodesic simplex in $S^{n}$. We map each simplex of $T$ to the corresponding geodesic simplex. This defines a retraction of $T$ and proves the inequality Fill $\operatorname{Rad} S^{n} \geqslant \frac{1}{2} L\left(S^{n}\right)$.

Remarks. 1. The filling radius being defined for manifolds with an arbitrary metric (Riemannian or not), the same argument using Jung's theorem in the Euclidean case and Remark 1 following the proof of Theorem 1 shows that Fill $\operatorname{Rad} S^{n} E=\frac{1}{2} L\left(S^{n} E\right)=\frac{1}{2} \sqrt{2+2 /(n+1)}$.

2. Suppose $S^{n}$ is imbedded in a metric space $T$ which is not triangulable, and let $R=\frac{1}{2} L\left(S^{n}\right)$. If $T$ is contained in the open $R$-neighborhood of $S^{n} \subset T$, the retraction may be constructed as follows. Given a point $x \in T$, consider the center of mass in $\mathbf{R}^{n+1}$ of the set $\left\{s \in S^{n} \mid \operatorname{dist}(x, s) \leqslant R\right\}$ weighted by the function $R-\operatorname{dist}(x, s)$. By Jung's theorem, the center of mass is not the origin in $\mathbf{R}^{n+1}$. Then its radial projection to $S^{n}$ gives the image of $x \in T$ under the retraction.

\section{Alexandrov-Toponogov theorem and estimates for $\mathbf{C} P^{n}, \mathbf{H} P^{n}, C a P^{2}$}

Suppose a Riemannian manifold $X$ has sectional curvature bounded above by +1 and injectivity radius at least $\pi$.

Lemma 3. Let bcd be a triangle in $X$ with sides of length $<L$, where $\pi / 2 \leqslant L \leqslant 2 \pi / 3$, and let $u$ be a point on the side $c d$. Then $\operatorname{dist}(b, u)$ is less than the height of the equilateral spherical triangle with side $L$ in $S^{2}$.

Proof. The perimeter of each of the triangles $c u b$ and $d u b$ is less than $2 \pi$, and therefore the triangles lie within the injectivity radius of the point $u$. Choose points $B, C, D, U$ in $S^{2}$ so that triangles $C U B$ and $D U B$ have sides equal to the corresponding sides of $c u b$ and $d u b$, and so that $C$ and $D$ are separated by the great circle containing $B$ and $U$. The Alexandrov-Toponogov comparison theorem (see [4, Theorem 2.7.6, p. 219]) asserts that the angles of $C U B$ and $D U B$ are no less than the corresponding angles of $c u b$ and $d u b$. In particular, $\angle C U B+\angle D U B \geqslant \pi$. Extend the arc $B U$ beyond $U$ until it reaches at point $U^{\prime}$ the shortest arc joining $C$ and $D$. Then we show that

$$
\operatorname{dist}\left(B, U^{\prime}\right)=\operatorname{dist}(B, U)+\operatorname{dist}\left(U, U^{\prime}\right) .
$$


Suppose that the extended arc does not realize the distance between $B$ and $U^{\prime}$, and let $B^{\prime}$ be the point of the sphere opposite $B$. Then $B^{\prime}$ lies on $U U^{\prime}$ and in the interior of the triangle $C U D$, and therefore

$$
\operatorname{dist}\left(B^{\prime}, C\right)+\operatorname{dist}\left(B^{\prime}, D\right) \leqslant \operatorname{dist}(U, C)+\operatorname{dist}(U, D)<L .
$$

This means that the length of the closed curve consisting of the $\operatorname{arcs} B^{\prime} C, C B$, $B D, D B^{\prime}$ is $<3 L \leqslant 2 \pi$ which is impossible. The contradiction shows that $\operatorname{dist}(b, u) \leqslant \operatorname{dist}\left(B, U^{\prime}\right)$, and the proof is reduced to the trivial case $X=S^{2}$.

Remark. In fact we proved that if $\beta \gamma \delta$ is a triangle in $S^{2}$ with sides equal to the corresponding sides of $b c d$, then there is a point on $\gamma \delta$ with distance from $\beta$ greater than or equal to $\operatorname{dist}(b, u)$.

Corollary. Let $a, b, c, d$ be four points in $X$ with pairwise distances less than the spherical distance between two vertices of a regular tetrahedron inscribed in $S^{2}$. Let $u$ be a point on $c d$, and $v$, a point on bu. Then $\operatorname{dist}(a, v)<\pi$, so that $a$ and $v$ are joined by a unique shortest arc.

Proof. Apply Lemma 3 and the obvious correlation $L+2 H=2 \pi$, where $L$ is the distance between vertices, and $H$ the height of a 2 -face of the tetrahedron.

Let $\mathbf{C} P^{n}$ be the $n$-dimensional complex projective space with its canonical metric, such that every complex projective line $\mathbf{C} P^{1} \subset \mathbf{C} P^{n}$ is a sphere $S^{2}$ of curvature +1 . We have Fill $\operatorname{Rad} C P^{1}=\frac{1}{2} \arccos \left(-\frac{1}{3}\right)$ (Theorem 2).

Proposition 1. The filling radius of $\mathbf{C} P^{n}$ is greater than or equal to that of C $P^{1}$ :

$$
\text { Fill } \operatorname{Rad} C P^{n} \geqslant \text { Fill } \operatorname{Rad} C P^{1}=\frac{1}{2} \arccos \left(-\frac{1}{3}\right) \text {. }
$$

Proof. Recall that the sectional curvature $K$ of $\mathbf{C} P^{n}$ satisfies $\frac{1}{4} \leqslant K \leqslant 1$ [1, p. 73], and that Inj $\operatorname{Rad} C P^{n}=\pi$.

Every ordered 4-tuple of points $\{a, b, c, d\}$ in $\mathbf{C} P^{n}$ with pairwise distances $<\arccos \left(-\frac{1}{3}\right)$ spans a standard 3-simplex in $\mathbf{C} P^{n}$, constructed as follows. We join $b$ with all points of the edge $c d$, producing the face $b c d$, and then join $a$ with all points of $b c d$ by shortest arcs. The simplex is well defined by the preceding corollary.

To prove the proposition, it suffices to retract to $\mathbf{C} \boldsymbol{P}^{n}$ every given polyhedron $T$ of real dimension $2 n+1$, contained in the open neighborhood of $\mathbf{C} P^{n} \subset L^{\infty}\left(\mathbf{C} P^{n}\right)$ of radius $\frac{1}{2} \arccos \left(-\frac{1}{3}\right)$ (see the proof of Theorem 2). We send each vertex of $T$ to a nearest point of $\mathbf{C} P^{n}$. By taking a sufficiently fine triangulation of $T$ we may assume that the endpoints of every edge of $T$ are sent to points with distance $<\arccos \left(-\frac{1}{3}\right)$. We choose a fixed ordering of the vertices of $T$. This induces an ordering on the collection of vertices of each 
simplex of $T$. We retract each 3-simplex to the corresponding standard simplex in $\mathbf{C} P^{n}$. Since the homotopy groups $\pi_{i}\left(\mathbf{C} P^{n}\right), i=3, \ldots, 2 n$, are trivial (use the exact sequence of the Hopf fibration), the retraction extends to the entire polyhedron.

Proposition 2. Let $\mathbf{H} P^{n}$ be the quaternionic projective space, and $\mathrm{Ca} \mathrm{P}^{2}$ the Cayley plane. Then

$$
\begin{aligned}
& \text { Fill } \operatorname{Rad} \mathbf{H} P^{n} \geqslant \frac{1}{2} \arccos \left(-\frac{1}{5}\right), \\
& \text { Fill } \operatorname{Rad} \mathrm{Ca} P^{2} \geqslant \frac{1}{2} \arccos \left(-\frac{1}{9}\right) .
\end{aligned}
$$

Proof. Define the real numbers $H_{i}>0, i=0,1,2,3$, recursively as follows. Set $H_{0}=L\left(S^{4}\right)=\arccos \left(-\frac{1}{5}\right)$. Consider the isosceles triangle in $S^{2}$ with base of length $H_{0}$ and sides $H_{i}$. Define $H_{i+1}$ to be the length of the perpendicular dropped from one of the vertices of the base to the opposite side of the triangle. Then the correlation

$$
\text { (+) } \quad H_{0}+2 H_{3}=2 \pi
$$

is equivalent to the following description of the regular inscribed 5-simplex in $S^{4}$.

Fix a 3-face of the simplex. Let $A$ and $B$ be the two remaining vertices, and let $C$ be the center of the fixed 3-face. Then $A, B$, and $C$ lie on a common great circle. The point $C$ is the farthest from $B$ among all the points of the fixed 3-face.

Every ordered 6-tuple $\left\{a_{0}, \ldots, a_{5}\right\}$ of points in $\mathbf{H} P^{n}$ with pairwise distances less than $H_{0}$ spans a standard 5-simplex constructed by joining $a_{i}$ with every point of the (i-1)-face $a_{0} \cdots a_{i-1}$, where $i$ runs from 1 to 5 . That the simplex is well defined is immediate from $(++)$ and the remark following Lemma 3.

Let $T \subset L^{\infty}\left(\mathbf{H} P^{n}\right)$ be a polyhedron with sufficiently small simplices contained in the $\left(\frac{1}{2} H_{0}\right)$-neighborhood of $\mathbf{H} P^{n}$. We send each vertex of $T$ to a nearest point of $\mathbf{H} P^{n}$, and then construct the retraction $r: T^{5} \rightarrow \mathbf{H} P^{n}$ of the 5-skeleton $T^{5} \subset T$ by sending each 5-simplex to the corresponding standard simplex in $\mathbf{H} P^{n}$.

Let $f: \mathbf{H} P^{n} \rightarrow K\left(\mathbf{Z}_{2}, 4\right)$ be a map into the Eilenberg-MacLane space such that the induced homomorphism on 4-dimensional cohomology with coefficients in $\mathbf{Z}_{2}$ sends the generator $a$ of the group $H^{4}\left(K\left(\mathbf{Z}_{2}, 4\right)\right) \simeq \mathbf{Z}_{2}$ to the generator $b$ of the group $H^{4}\left(\mathbf{H} P^{n}\right) \simeq \mathbf{Z}_{2}, f^{*}(a)=b$.

The composition $f \circ r: T^{5} \rightarrow K\left(\mathbf{Z}_{2}, 4\right)$ extends to a mapping $g: T \rightarrow K\left(\mathbf{Z}_{2}, 4\right)$. We will show that $g_{*}\left(\left[\mathbf{H} P^{n}\right]\right) \neq 0$, where $\left[\mathbf{H} P^{n}\right]$ is the fundamental homology class of $\mathbf{H} P^{n}$.

Since $g=f$ on the 4-skeleton, we have $g^{*}(a)=f^{*}(a)=b$. Recall that the fundamental cohomology class of $\mathbf{H} P^{n}$ equals the cup product power $b^{n}$ of $b$. 
Using a natural pairing, we write

$$
\begin{aligned}
1 & =\left\langle b^{n},\left[\mathbf{H} P^{n}\right]\right\rangle=\left\langle\left(g^{*}(a)\right)^{n},\left[\mathbf{H} P^{n}\right]\right\rangle \\
& =\left\langle g^{*}\left(a^{n}\right),\left[\mathbf{H} P^{n}\right]\right\rangle=\left\langle a^{n}, g_{*}\left(\left[\mathbf{H} P^{n}\right]\right)\right\rangle,
\end{aligned}
$$

and therefore $g_{*}\left(\left[\mathbf{H} P^{n}\right]\right) \neq 0$.

The case of $\mathrm{CaP} P^{2}$ is treated similarly.

Proposition 3. The filling radius of every simply connected two-point homogeneous space (namely, $\mathrm{S}^{n}, \mathbf{C} P^{n}, \mathbf{H} P^{n}$, or $C a P^{2}$ ) is strictly less than one third of its diameter.

Proof. The above assertion is true for the spheres (Theorem 2). We will exhibit a number $\varepsilon_{n}>0$ such that Fill $\operatorname{Rad} C P^{n} \leqslant \pi /\left(3+\varepsilon_{n}\right)$; the other two cases are treated similarly.

Consider the Hopf fibration of the unit $(2 n-1)$-sphere in $\mathbf{C}^{n}$. One may construct a closed subset $Z_{n}$ of this sphere out of a sufficient number of small balls, such that $Z_{n}$ contains no antipodal points and meets every semicircle of every fiber. Define $\varepsilon_{n}$ by $\left(1+\varepsilon_{n}\right)$ diam $Z_{n}=2$, where diam is the Euclidean diameter. We identify $\mathbf{C}^{n}$ with the tangent space to $\mathbf{C} P^{n}$ at a fixed point $x$, and define the subset $Y_{n} \subset \mathbf{C} P^{n}$ by

$$
Y_{n}=\exp \frac{\pi\left(1+\varepsilon_{n}\right)}{3+\varepsilon_{n}} Z_{n} .
$$

Since $C P^{n}$ is positively curved, we have $\operatorname{diam} Y_{n}<2 \pi /\left(3+\varepsilon_{n}\right)$. Take an arbitrary point $x^{\prime} \in \mathbf{C} P^{n}, x^{\prime} \neq x$, and let $\mathbf{C} P^{1}$ be the unique complex projective line passing through $x$ and $x^{\prime}$. Our assumption on $Z_{n}$ implies that $\operatorname{dist}\left(x^{\prime}, Y_{n} \cap \mathbf{C} P^{1}\right) \leqslant 2 \pi /\left(3+\varepsilon_{n}\right)$. Hence $Y_{n}$ satisfies the conditions of Lemma 1 with $R=\pi /\left(3+\varepsilon_{n}\right)$.

\section{References}

[1] J. Cheeger \& D. G. Ebin, Comparison theorems in Riemannian geometry, North-Holland, Amsterdam, 1975.

[2] H. Federer, Geometric measure theory, Springer, Berlin, 1969.

[3] M. Gromov, Filling Riemannian manifolds, J. Differential Geometry 18 (1983) 1-147.

[4] W. Klingenberg, Riemannian geometry, de Gruyter, Berlin, 1982.

Université Paris VI, Paris 
